\title{
Physical Examination Indicated Rescue Cerclage to Improve Live Birth Rate at Hung Vuong Hospital in Vietnam
}

\author{
Huynh Nguyen Khanh Trang1,2*, Tran Thi Ngoc Tam², Pham Huynh Phuc Hung2, \\ Hoang Thi Diem Tuyet ${ }^{1,2}$ \\ ${ }^{1}$ Pham Ngoc Thach University of Medicine, HCMC, Vietnam \\ ${ }^{2}$ Hung Vuong Hospital, HCMC, Vietnam \\ Email: *tranghnk08@gmail.com,ngoctam237@gmail.com,phphuchung@gmail.com, tuyethoang05@yahoo.com.vn
}

How to cite this paper: Trang, H.N.K., Tam, T.T.N., Hung, P.H.P. and Tuyet, H.T.D. (2020) Physical Examination Indicated Rescue Cerclage to Improve Live Birth Rate at Hung Vuong Hospital in Vietnam. Open Journal of Obstetrics and Gynecology, 10, 400-408.

https://doi.org/10.4236/ojog.2020.1030036

Received: February 11, 2020

Accepted: March 23, 2020

Published: March 26, 2020

Copyright $\odot 2020$ by author(s) and Scientific Research Publishing Inc. This work is licensed under the Creative Commons Attribution International License (CC BY 4.0).

http://creativecommons.org/licenses/by/4.0/

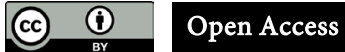

\begin{abstract}
Emergency cerclage with advanced cervical dilatation and bulging of fetal membranes is still challenging to obstetricians. Objective: To identify the live birth rate after physical examination indicated rescue cerclage. Methods: A retrospective cohort study of 36 pregnant women, who underwent rescue cerclage at Hung Vuong hospital from January 2015 to January 2019. Selection criteria: cervical dilation 10 to $40 \mathrm{~mm}$, at 20 to 30 gestational weeks. Exclusion criteria: multiple pregnancy, birth defects, pregnancy termination due to maternal or fetal indications. Results: Delivery of live infant rate was $72.22 \%$; mean pregnancy prolongation was $42.8 \pm 34.15$ days. Early live birth rate was $77.78 \%$. The percentage of delivery after 34 and 37 weeks was $44.45 \%$ and $27.78 \%$, respectively. Live birth weight was 400 to $3600 \mathrm{~g}$, with mean being $1878.06 \pm 1049.04$ g. No procedure-related complication was observed. Conclusions: Rescue cerclage may be an effective and safe method in prolonging pregnancy and improving neonatal outcome in women with cervical dilation 10 to $40 \mathrm{~mm}$. It should be considered as a viable option for women with a dilated cervix in 20 to 30 gestational weeks. Future controlled studies are needed.
\end{abstract}

\section{Keywords}

Emergency Cerclage, Delivery of Live Infant Rate

\section{Introduction}

Cervical insufficiency is an incomplete condition of the uterus cervix, resulting in extremely premature birth or late abortion in the absence of signs of uterine 
contraction [1] occurring in $1 \%$ of the population and $8 \%$ in cases of recurrent pregnancy loss in the second trimester of pregnancy [2]. Cervical insufficiency can lead to extremely premature birth, leading to increased neonatal mortality and morbidity. Therefore, cervical cerclage with cervical insufficiency is a practical issue in pregnancy management.

The use of cervical cerclage in the prevention of preterm delivery was described by Shirodkar in 1955 and then by McDonald two years later. It is not clear why dilatation and effacement of the cervix occur prematurely, but it is thought that the forced mechanical closure of an "incompetent" cervix with a suture maintains the cervical length as well as the mucus plug-both of which have a role in preventing labour. However, there is a lack of good large randomized controlled trials to help clinicians and patients decide whether or not to insert a cervical suture-the three main randomized controlled trials having conflicting results. Cervical cerclage may be performed prophylactically in the first trimester when the clinical history suggests risk of mid-trimester loss or when cervical resistance studies confirm low cervical resistance. It may also be performed when there is evidence of a short cervix $(<25 \mathrm{~mm})$ or cervical shortening on ultrasound. More rarely, emergency cerclage, a rescue cervical suture may be inserted when the pregnant women present with a cervix that is already advanced dilitation with the membranes bulging into the vagina but no signs of labour, infection or heavy vaginal bleeding [3].

Once the cervix is open, stitching the cervix is one of the effective methods to help prolong gestation age. Aoki et al. 2014 study showed an average of gestation length up to 44 days after cervical suture (the shortest is 4 days and the longest is 165 days). Whereas, the average of gestation length in the non-sutured group is 12.5 days, the shortest is 2 days and the longest is 93 days [4]. A retrospective study in China of 10 years on 158 cases of suturing when the cervix was open, and the amniotic sac was seen, showed that this technique helped prolonging pregnancy by an average of $52.16 \pm 6.2$ days, with the infant survival rate is $82.28 \%$ [5]. In comparison, the duration of extra pregnancy in a study in the UK was 42 days, but $33 \%$ patients having rupture of membranes as the procedure complication [3].

To contribute to assessing the effectiveness and safety of cervical cerclage when the cervix is open, we conducted a study with the question "How effective and safe is emergency cervical cerclage?". The aim of this study was to look retrospectively at the patients undergoing emergency cervical cerclage. This information may help the patient and her career make an informed decision about whether to undergo emergency procedure.

\section{Objects and Research Methods}

Design: retrospective cohort study.

Selection criteria: 36 single fetal pregnant women, with their cervix dilation from 10 to $40 \mathrm{~mm}$. Gestational age is from 20 weeks to less than 30 weeks. There 
was no uterine contraction at the time of cervical cerclage, no amniotic fluid leakage or suspected infection.

Exclusion criteria: multiple pregnancy, birth defects, cases of pregnancy termination due to maternal pathology (severe pre-eclampsia...) or fetal pathology (intrauterine growth retardation, fetal infection...).

Time: cases of importing with the diagnosis of extremely preterm labor or threatened preterm labor with a cervical opening from 10 - $40 \mathrm{~mm}$ at Hung Vuong hospital from January 1, 2015-January 1, 2019.

+Before performing emergency cerclage: cervical opening status by manual examination with speculum as well as vaginal ultrasound evaluation to assess the level, shape, funnel formation of amniotic fluid sac. Nitrazine test was performed to eliminate amniotic fluid leakage. All patients received progesterone $100 \mathrm{mg}$ to $200 \mathrm{mg} /$ day. $\mathrm{MgSO}_{4} 36$ hours intravenously for the purpose of protecting the fetal brain.

Evaluation of infection with leukocytes (over $11,000 / \mathrm{mm}^{3}$ ) in total blood cell analysis, C-reactive protetin (over $10 \mathrm{mg} / \mathrm{L}$ ) and serum procalcitonin $(0.05 \mathrm{ng} / \mathrm{mL}$ ).

Using Bethamesone intramuscularly $12 \mathrm{mg}, 2$ times 24 hours to stimulate maturity of the fetus spectrum at 24 weeks or more.

Prophylactic antibiotics were indicated for all cases with Cefazolin $1 \mathrm{~g}$ by slow intravenous injection 30 minutes before the procedure.

Low-dose spinal anesthesia was considered as insensitive at the procedure.

The bladder was emptied before the procedure in all cases.

+After emergency cerclage: reduce the uterus contractions at least $24-48$ hours after the procedure. The patient was discharged after emergency cerclage along with no 48-hour uterus contractions and complications such as amniotic rupture, bleeding ... The sutures were cut at 37 weeks gestation or during labor, as well as amniotic rupture before 37 weeks.

\section{Result}

All 36 participants followed the study were analysed. The age mean of participants is $27.03 \pm 5.21$ years old. Among participants, $24 / 36$ (66.67\%) were nulliparity. Almost all of them got pregnancy naturally, only $3 / 36(8.33 \%)$ from Assisted reproductive technology. 11/36 study subjects (30.55\%) had cerclage at previous pregnancise but only one case (2.78\%) had History of extremely premature birth. 36 study participants were identified cervical insufficiency with physical examination. All of them underwent rescue cerclage at Hung Vuong hospital from January 2015 to January 2019.

After rescue cerclage, we confirm successful cases when the pregnancies prolonged more than 48 hours. This duration was enough until the possible manifestation of corticosteroid administration effect.

Besides evaluation the outcomes relating to mother, our study also concerned the outcome relating the new born babies. In our study, the live birth rate was $77.78 \%$ (28/36), among them, 26 (72.22\%) alive babies were discharged. 


\section{Discussion}

During the period from January 1, 2015 to January 1, 2019, our study recorded 36 emergency cerclage cases with all the research conditions.

The median age of pregnant women was $27.03+/-5.21$ years, of which the group of pregnant women of reproductive age accounted for $91.67 \%$ (Table 1).

The rate of prolonging pregnancy at least 48 hours is $94.44 \%$ (Table 2), enough time for steroides to act on small cells in the alveoli of the lungs to produce surfactant. The opening of the cervix before suturing ranges from $10 \mathrm{~mm}$ to a maximum of $40 \mathrm{~mm}$. Through exam by speculum, we recorded 21 (58.33\%) cases with associated bulging of fetal membraches at time of cerclage cervical. The time from admission to cervical cerclage is about 4 days (Table 1). Cases of emergency cerclage when the gestational age was less than 24 weeks accounted for $30.56 \%$, as early as 20.5 weeks, the oldest gestational age in our study with emergency cervical cerclage was 30 weeks (1 woman).

Emergency cervical cerclage has been applied in many parts of the world with the aim of prolonging pregnancy, increasing live birth rate. Our study recorded a success rate of $94.44 \%$ for emergency cerclage with an average gestation prolongation from cervical cerclage to birth of 42.8 days (Table 2). In particular, the

Table 1. Characteristics of research subjects $(n=36)$.

\begin{tabular}{cc}
\hline Factor & $\mathbf{n}(\%)$ \\
\hline Gestational age at admission (week) & $24.9 \pm 2.58$ \\
The average number of cervical openings $(\mathrm{mm})$ & $17.7 \pm 9.92$ \\
Cervical openings $>10 \mathrm{~mm}-<20 \mathrm{~mm}$ & $16(44.4)$ \\
Cervical openings $<20 \mathrm{~mm}$ & $5(13.9)$ \\
Cervical openings $<30 \mathrm{~mm}$ & $3(8.4)$ \\
Cervical openings $\leq 40 \mathrm{~mm}$ & $13.17 \pm 5.25$ \\
Average cervical lengths (mm) & $21(58.33)$ \\
Bulging of fetal membranes (yes) & $3(8.33)$ \\
Uterine activity before cervical cerclage & $25.56 \pm 2.53$ \\
\hline
\end{tabular}

Table 2. Outcome of rescue cervical cerclage.

\begin{tabular}{cc}
\hline Outcomes & $\mathbf{n}(\%)$ \\
\hline The number of pregnancies lasted more than 48 hours & $34(94.44)$ \\
Average time from emergency cervical to birth (days) & $42.8 \pm 34.15(1.37-105)$ \\
Uterine activity after emergency cerclage & $9(25)$ \\
Complications when performing emergency cerclage & 0 \\
\hline
\end{tabular}


proportion of children alive at discharge from hospital is $72.22 \%$ (26/36 cases Table 3).

Our study calculated the proportion of children alive at discharge by dividing the total number of children with cure to the total number of eligible pregnant women enrolled in the study. Serious cases of death are considered as death. The rate of alive children at discharge in our study is $72.2 \%$, higher than its in the study of Gundabattula [6] (50.7\%). The difference may be due to the study of Gundabattula applying all cases with cervix opening from 10 up to $60 \mathrm{~mm}$, while our study only selected cases with the cervix opening from $10 \mathrm{~mm}$ to $40 \mathrm{~mm}$. Regarding the gestational age at birth, our birth rate before 34 weeks is $44.45 \%$. This result is similar to that of Shivani [7] (42.5\%) and lower than that of Gundabattula [6] (69.5\%). Differences between the selection criteria may be the cause of differences in the results between our study and other authors. Given the limitations of extremely preterm and preterm neonatal care in Vietnamese hospitals, extending the pregnancy to 34 weeks is particularly significant. According to the Royal College of Obstetricians and Gynaecologists, cervical stitches provide relief by an average of 5 weeks longer for pregnant women than for non-sutured groups; there is a correlation with a reduction in the rate of births before 34 weeks in the cervical suture group [8]. A randomized controlled trial of Althuisius SM et al. (2003) was performed to compare the effectiveness between cervical suture and bed rest in 23 patients at gestational age 22 - 23 weeks . Pregnant women in the cervical suture group had a longer gestation period than the group without cervical suture on average 4 weeks (54 days compared to 20 days) and the rate of preterm birth before 34 weeks decreased significantly: $53 \%$ and $100 \%$, with p-value: $<0.02)$ [9].

All of cases in our study were sutured successful. We have not recorded any cases of amniotic rupture or heavy bleeding at cervical cerclage preocedure.

Table 3. Neonatal outcomes after birth and postpartum.

\begin{tabular}{cr}
\hline Factors & Average (SD)/n (\%) \\
\hline Newborn live at birth & $28(77.78)$ \\
Alive babies discharged & $26(72.22)$ \\
Apgar score in 1 minute < 5 & $1878.06 \pm 1049.04$ \\
Apgar score in 5 minutes < 5 & $14(38.89)$ \\
Bethamethasone, stimulating lung maturation & $14(38.89)$ \\
Admission at neonatal department & $22(62.89)$ \\
Length of time in neonatal department (Median, days) & $17(47.22)$ \\
Admission NICU & $4(0-36)$ \\
Length of time in NICU (Median, days) & $15(41.67)$ \\
Tracheal surfactant pump & $11(0-58)$
\end{tabular}

SD: Standard deviation; NICU: Neonatal intensive care unit. 
There were 2 cases of preterm labor, with cervix tearing and two of them were sewn to recover after that. Author Zhu [5] (2015) also recorded two cases of cervical tear in his study. However, this author also noted some more serious adverse effects such as pulmonary edema or deep vein embolism. Meanwhile, Fuchs' research [10] showed that there were 2 cases of amniotic sac rupture right at the time of suturing, leading to miscarriage. The lack of a control group makes it difficult to determine whether rescue cervical cerclage was detrimental or not.

Once the cervix is open, the amniotic sac in contact with the vagina is at risk of infection. Our study carried out the pre-septic infection assessment with routine testing of white blood cells, serum C-reactive protein and serum Procalcitonin. Hashim [11] argues that there is insufficient evidence to recommend routine pre-treatment of leukocyte and C-reactive proteins in order to detect subclinical infections early on. A number of studies have shown an association between increased C-reactive protein and the risk of preterm birth or premature leakage, but because the sensitivity and specificity of C-reactive protein are low, the use of these parameters should be considered in each specific case.

Gundabattula [6] reported 12 cases (57.1\%) with histological evidence of postpartum amnioticitis. In the study of Steenhaut [12], the rate of amniotic membranes rupture in alive children was $21.3 \%$. This ratio in the study of Curti et al. [13] is $30.7 \%$. Only 12 cases in the Steenhaut [12] study did not have amniotic membrane rupture, the remaining cases reported contact of the amniotic membrane to the vaginal environment.

Our study routinely tests white blood cells for all patients admitted to the hospital. C-reactive protein and Procalcitonin are taken when signs suggest a clinical infection: fever, tachycardia, ... Transplantation of vaginal or cervical fluid is only performed when suggestive of vaginitis. Before decision of conducting cervical cerclage, all patients were examined vaginally to identify cervical condition, amniotic membrane and perform nitrazine test. The follow-up is similar to the previous stitch.

In this study, we did not record any clinical symptoms of amniotic fluid infection before and after cervical suture. However, we did not conduct regularly infection markers pre- and post-procedure for all participants, we were currently unable to conclude the relationship between infection status and outcomes: new-born.

The opening of the cervix before suturing of the women in our study averaged $17.7 \pm 9.92 \mathrm{~mm}$ (Table 1 ). This result is similar to the result from the study of the authors Gundabattula [6]. However, Zhu's 2015 study [5] of the cervix was on average larger than our study. The first round neck emergency medical cervical sewing has only started at Hung Vuong Hospital in the last 5 years. Differences in experience accumulated over time as well as improvements in methods can lead to different patient selection decisions.

Circulation, when the cervix has been opened, has many risks such as: rupture of the amniotic mambranes, creating uterine contractions causing premature 
birth, cervical tear or uterine rupture. Therefore, it is necessary to make a definite diagnosis of cervical openness due to uterine open waist, which excludes preterm labor. Recently, we combined using the Parto-Sure test to find Placental alpha microglobulin-1 (PAMG-1) at the same map to help increase the prognosis for success when implementing emergency cerclage. At Hung Vuong Hospital, at present, we only apply cervical suture when the opening is $\leq 40 \mathrm{~mm}$. Cases of cervical opening $>40 \mathrm{~mm}$ often accompanied by painful uterine contractions, regularly. All of these cases then went into preterm labor within 48 hours.

The Royal College of Obstetricians and Gynaecologists (2011) guidelines suggest that cervical openings more than $40 \mathrm{~mm}$ wide or the presence of amniotic fluid in the vagina is one of the prognostic factors for failure of cervical suture. However, because there are insufficient data from controlled studies, it is not possible to conclude a causal relationship between cervical openness and failure [8].

Our study did not collect data from multiple pregnancies. The role of cervical cerclage stitching in multiple pregnancies remains controversial. In particular, in cases when the cervix is opened, cervical stitching is almost the only treatment option (compared to absolute bed rest). Although no randomized control trial study has been performed specifically on multiple pregnancies, the results from retrospective studies show that cervical lifecycle in multiple pregnancies helps prolong pregnancy by 7.3 days to 91 days [7] [14] [15]. In the trend of increasing fertility support, the rate of multiple pregnancies is also increasing. Therefore, there is a need for future studies to assess the role and safety of cervical lifecycle in this patient group.

Results of multivariate analysis of gestational age were associated with the proportion of infants born at discharge (Table 4). This result is also consistent with the research results of Gundabattula [6].

\section{Conclusion}

Emergency cerclage indicated cervical cerclage appears to have low complication rates and high live-birth rates. A large randomized controlled study is required to determine whether this intervention actually prolongs pregnancy.

Table 4. Multivariate regression analysis of factors related to infant survival.

\begin{tabular}{ccccc}
\hline Prognostic variables & Unit of comparison & OR & $95 \% \mathrm{CI}$ & P-value \\
\hline Lenght of cervical & $+1 \mathrm{~mm}$ & 1.56 & $0.84-2.9$ & 0.12 \\
Openning cervical & $+1 \mathrm{~cm}$ & 0.9 & $0.74-1.09$ & 0.20 \\
UA before cerclage & Yes vs No & 0.76 & $0-174.2$ & 0.92 \\
UA after cerclage & Yes vs No & 0.11 & $0-16.13$ & 0.33 \\
GA when cerclage & +1 week & 4.74 & $1.77-29.21$ & 0.01 \\
Betamethasone & Yes vs No & 0.43 & $0-41.54$ & 0.71 \\
\hline
\end{tabular}

UA: Uterine activity. 


\section{Conflicts of Interest}

The authors declare no conflicts of interest regarding the publication of this paper.

\section{References}

[1] American College of Obstatricians and Gynecologists (2014) Practice Bulletin No. 142: Cerclage for the Management of Cervical Insufficiency. Obstetrics \& Gynecology, 123, 372-379. https://doi.org/10.1097/01.AOG.0000443276.68274.cc

[2] Alfirevic, Z. (2017) Cervical Stitch (Cerclage) for Preventing Preterm Birth in Singleton Pregnancy. Cochrane Database of Systematic Reviews, No. 6. https://doi.org/10.1002/14651858.CD008991.pub3

[3] Liddiard, A. (2011) Elective and Emergency Cervical Cerclage and Immediate Pregnancy Outcomes: A Retrospective Observational Study. JRSM Short Reports, 2, 91. https://doi.org/10.1258/shorts.2011.011043

[4] Aoki, S. (2014) Emergency Cerclage versus Expectant Management for Prolapsed Fetal Membranes: A Retrospective, Comparative Study. Journal of Obstetrics and Gynaecology Research, 40, 381-386. https://doi.org/10.1111/jog.12207

[5] Zhu, L.Q. (2015) Effects of Emergency Cervical Cerclage on Pregnancy Outcome: A Retrospective Study of 158 Cases. Medical Science Monitor, 21, 1395-1401. https://doi.org/10.12659/MSM.893244

[6] Gundabattula, S.R. (2013) Outcomes of Pregnancy in Women Who Had Rescue Cerclage for Cervical Insufficiency: A Single-Center Retrospective Study. Journal of Obstetrics and Gynaecology Research, 39, 1293-1300. https://doi.org/10.1111/jog.12059

[7] Shivani, D. (2018) Does Rescue Cerclage Work? Journal of Perinatal Medicine, 46, 876-880. https://doi.org/10.1515/jpm-2017-0311

[8] Royal College of Obstatricians \& Gynaecologists (2011) Cervical Cerlage. Green-Top Guideline No. 60.

[9] Althuisius, S.M. (2003) Cervical Incompetence Prevention Randomized Cerclage Trial: Emergency Cerclage with Bed Rest versus Bed Rest alone. American Journal of Obstetrics and Gynecology, 189, 907-910. https://doi.org/10.1067/S0002-9378(03)00718-X

[10] Fuchs, F. (2012) Predictive Score for Early Preterm Birth in Decisions about Emergency Cervical Cerclage in Singleton Pregnancies. Acta Obstetricia et Gynecologica Scandinavica, 91, 744-749. https://doi.org/10.1111/j.1600-0412.2012.01386.x

[11] Abu Hashim, H. (2014) A Review of the Contemporary Evidence on Rescue Cervical Cerclage. International Journal of Gynecology \& Obstetrics, 124, 198-203. https://doi.org/10.1016/j.ijgo.2013.08.021

[12] Steenhaut, P. (2017) Retrospective Comparison of Perinatal Outcomes Following Emergency Cervical Cerclage with or without Prolapsed Membranes. International Journal of Gynecology \& Obstetrics, 137, 260-264.

https://doi.org/10.1002/ijgo.12144

[13] Curti, A. (2012) Exam-Indicated Cerclage in Patients with Fetal Membranes at or Beyond External OS: A Retrospective Evaluation. Journal of Obstetrics and Gynaecology Research, 38, 1352-1357. https://doi.org/10.1111/j.1447-0756.2012.01882.x

[14] Abbasi, N. (2018) Outcomes Following Rescue Cerclage in Twin Pregnancies. The Journal of Maternal-Fetal \& Neonatal Medicine, 31, 2195-2201. 
https://doi.org/10.1080/14767058.2017.1338260

[15] Han, M.N., et al. (2018) The Impact of Cerclage in Twin Pregnancies on Preterm Birth Rate before 32 Weeks. The Journal of Maternal-Fetal \& Neonatal Medicine, 32, 2143-2151. https://doi.org/10.1080/14767058.2018.1427719 